\title{
DIAMETRIC FRACTURES OF THE PELVIS
}

\author{
G. F. Dommisse, Pretoria, South Africa
}

My interest in the mechanism and injuries of the pelvis was roused when, during the course of dissections on the cadaver, it became necessary to remove the sacrum from in front. This at first appeared a difficult task, but it soon became apparent that the symphysis pubis was the key. Once the interpubic ligaments and the fibrocartilage had been severed, the pelvis opened out like the covers of a book and the sacro-iliac joints offered little resistance; in fact they acted as hinges upon which rotation took place. Hyperextension of the hips gave further appreciable assistance in gaining a wide diastasis at the symphysis.

\section{FUNCTIONAL ANATOMY}

Special dissections were made of the interpubic ligaments and they revealed the importance of the anterior interpubic ligament. Although not all text-books of anatomy stress its importance this ligament completely dwarfs in strength the superior and inferior interpubic ligaments. These appear to have no independent existence, being merely the superior and inferior portions of the great anterior ligament (Figs. 1 to 3). Histological examination of the interpubic ligaments (Fig. 4) shows dense interwoven collagen and no elastic tissue. The fibrils blend with those of the fibrocartilage of the interpubic disc in the midline, and with those of the periosteum of the pubis more laterally. The tensile strength of the ligament has not yet been accurately assessed, but its fibres resemble in structure those of the tendo calcaneus. The reason for the great strength of this ligament is the great demand made upon it. The effect of its disruption in the human being may be observed after the operation known as "subcutaneous symphysiotomy" in which the interpubic ligaments are divided in order to increase the diameter of the pelvic ring (Fig. 5). In essence, the ligament assures the integrity of a tie mechanism linking the halves of an arch of which the sacrum forms the summit and the lineae arcuatae of the ilia the haunches.

The body weight imposes a vertical load which is transmitted through the arch to the hips as a shearing force. This force on each side has both horizontal and vertical components; it is the horizontal element which is resisted by the tie-beam of the arch (Fig. 6). The thrust obviously varies with the position of the trunk and pelvis relative to the legs; from time to time compression stress is applied at the symphysis pubis, and this is adequately resisted by the symphysial fibrocartilage.

The pelvic arcade-The trabecular pattern of the pelvic arch is continued down into the calcar femorale (Fig. 7). Not only is there a central arch whose summit is formed on each side by the sacrum, but also this central arch is flanked by two additional arches formed by the side wall of the pelvis between the acetabulum and the anterior superior iliac spine, and by the ilio-tibial tract with the gluteus medius and minimus down to their femoral attachment. An arcade structure thus exists, perfect for carrying the load that the trunk imposes upon it, and for transmitting this load to the legs which are its columns.

The problem of the central arch is not quite so simple in man, because movements take place at the sacro-iliac joints; mobility as well as stability is, however, permitted by the introduction of strong interosseous and posterior ligaments which are placed at the positions of greatest stress, posteriorly and superiorly. Clearly, these ligaments are effective only so long as the tie-beam of the arch is intact. The moment it is disrupted (Fig. 5) the sacro-iliac joints must and do separate or widen in front. The anterior ligaments are too thin to offer any resistance to this diastasis. 
A special radiographic view of the sacro-iliac joints (Fig. 8) shows the extraordinary nature of the summit of the arch. Its taper is reversed, yet it retains its stability. It does so by virtue of three factors: 1) the concavo-convex shape of the opposing joint surfaces in every plane; 2) the small but effective " shelf " which is formed anteriorly by the iliac component of the sacro-iliac joint: this shelf is present over only a small segment of the sacro-iliac joint, where it acts to maximum advantage; and 3) the strong posterior and interosseous sacro-iliac ligaments that have been referred to above.

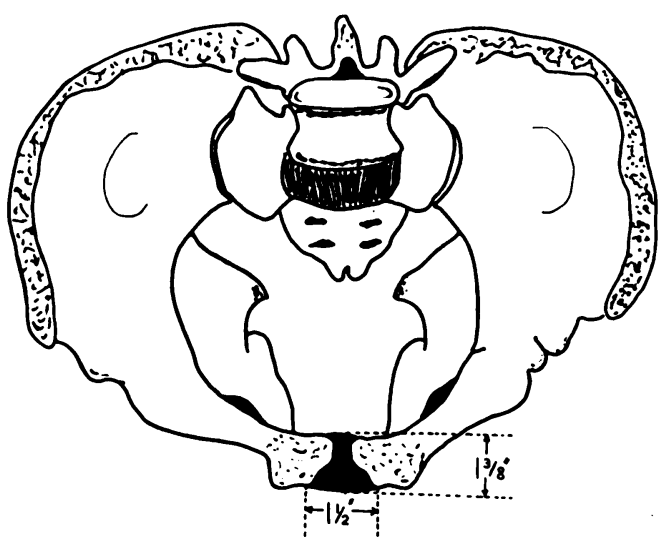

FIG. 1

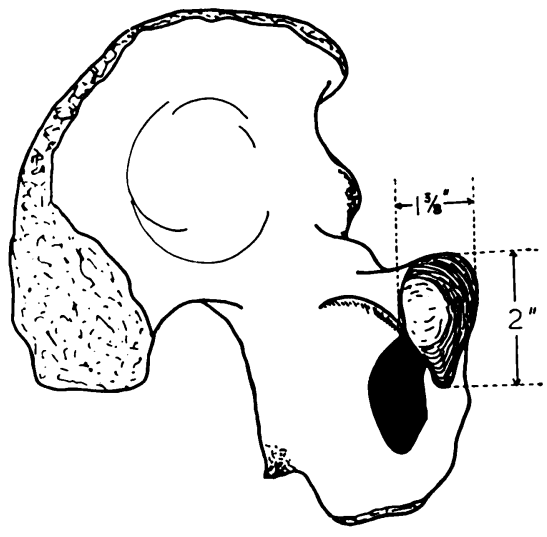

Fig. 2

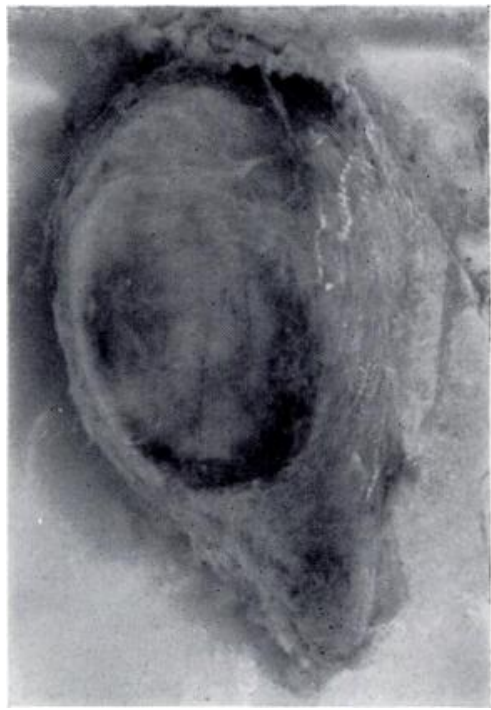

Fig. 3

The purpose of this odd and complicated structure may be to allow both mobility and stability at the sacro-iliac joints. Mobility and stability, being opposite qualities, can only be gained at a price; the price is the destruction of the articular cartilage of the sacro-iliac joints. In individuals over thirty years old the cartilage of the sacro-iliac joint appears radiographically to be only one-half or a third as thick as that of individuals sixteen to eighteen years old. The atrophic sacral articular cartilage commonly encountered after the third decade in men is shown in Figure 9.

VOL. 42 B, No. 3, AUGUST 1960 


\section{CLINICAL MATERIAL}

Forty cases have been selected for this survey. Trivial lesions such as avulsion of muscular attachments have been excluded. The earliest case in the series has been followed for twelve years.

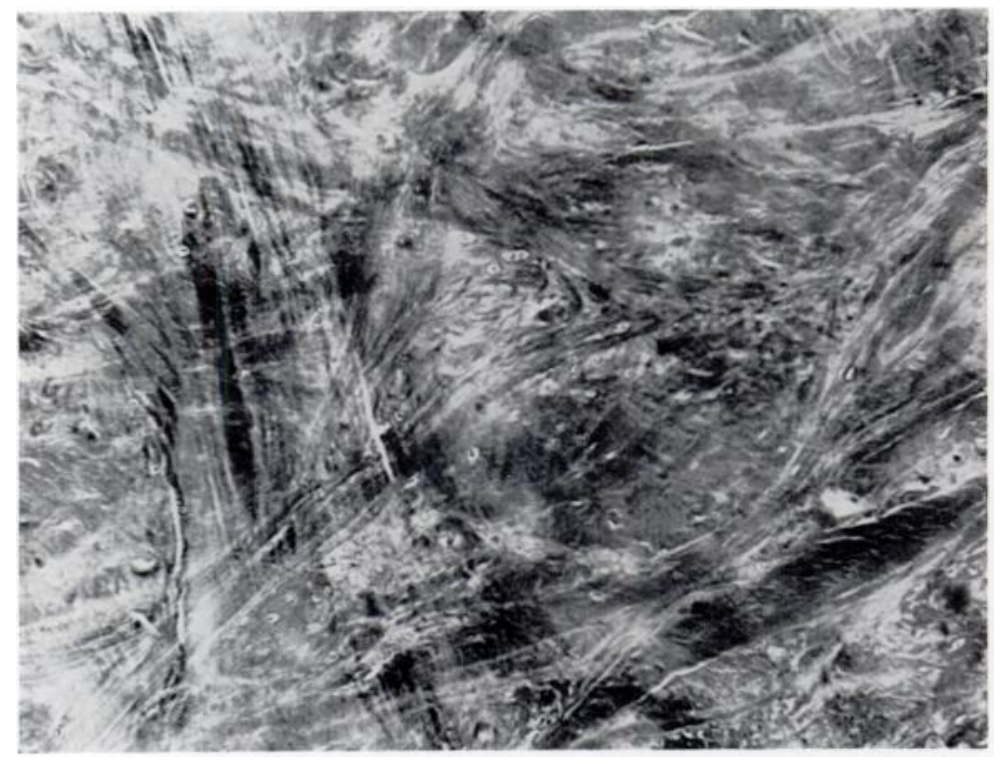

Fig. 4

Section of the anterior pubic ligament ( $\times 80$, stained haematoxylin and eosin) showing the interlacing collagen fibres.

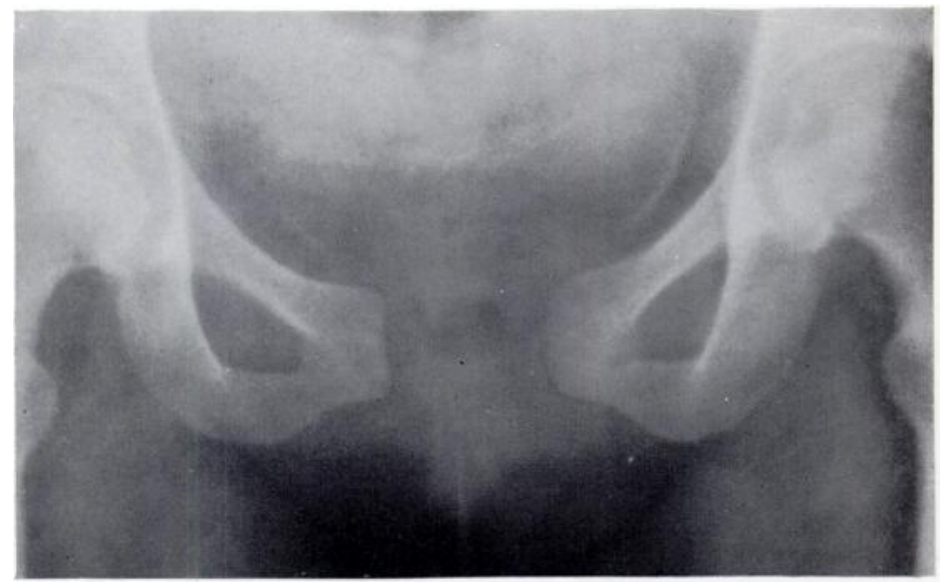

FIG. 5

Radiograph of symphysis pubis nine months after symphysiotomy. Note the wide separation of the pubic bones.

Classification-Classification is based upon the mechanism of the production of the injury; the fractures fall quite simply into two major groups: 1) fractures caused by violence transmitted to the pelvis, and 2) fractures caused by direct compression of the pelvis.

Fractures from transmitted violence-These are the common injuries and are sustained by the occupants of vehicles. The patient is injured sitting; the force of a blow on the flexed knee is transmitted to the pelvis along the shaft of the femur. Anything can happen to the pelvis, 
depending on the exact position of abduction or adduction of the hip, the degree of flexion and the strength and duration of the deforming force.

The basic lesion (Fig. 10) takes place when flexion of the hip is below a right angle, with neither abduction nor adduction at the hip. The shelf of the acetabulum resists the blow, and the interpubic ligaments and the anterior sacro-iliac ligaments are torn. The pattern may vary

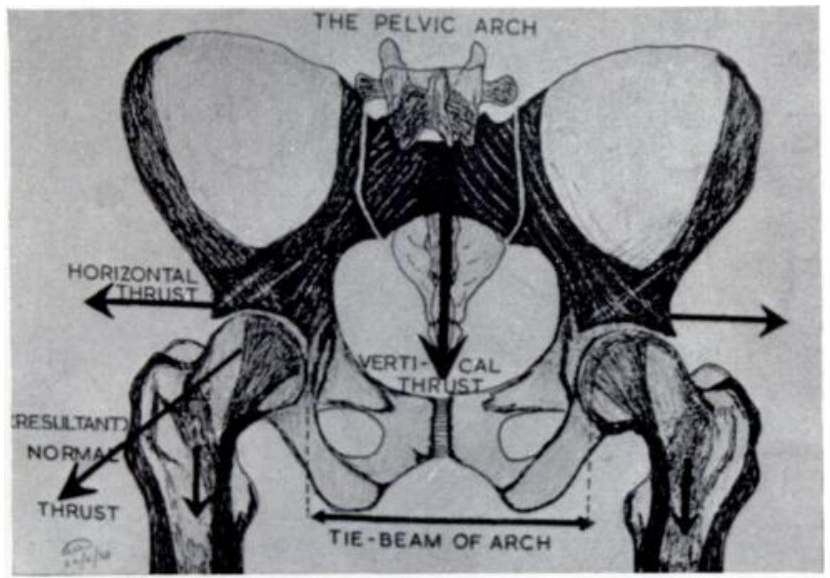

FIG. 6

The trabecular pattern of the pelvic arch; a diagram based on a study of several radiographs.

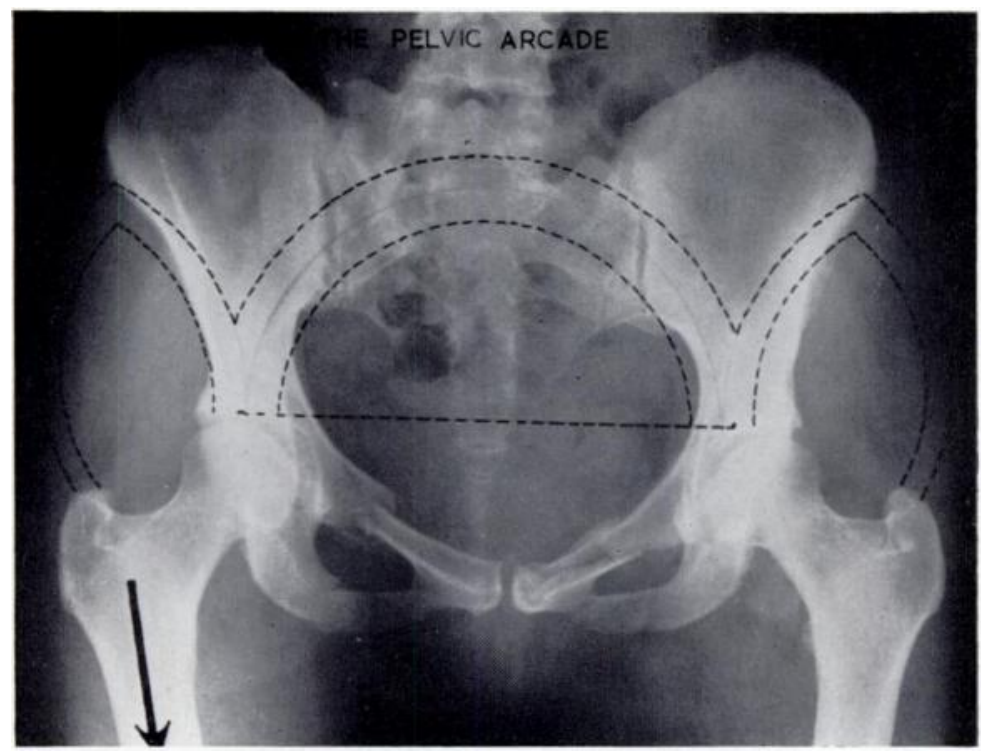

FiG. 7

Radiograph of pelvis, showing the "pelvic arcade."

inasmuch as a fracture through the pubic rami or through the wing of the ilium on one or other side may be encountered, while the ligaments remain intact. The mechanism of the lesion remains basically the same. Treatment is simple; displacement may be reduced by the technique of Watson-Jones (1955) and reduction maintained by a short double plaster spica. A pelvic sling, with or without traction on the legs, may fail to secure reduction because the position of extension of the hips acts as a continuing deforming factor. If, on the other hand, the patient

VOl. 42 B, No. 3, AUGUST 1960 
were to be nursed in the lateral position with the hips flexed there would be no need for manipulation or for splintage and a period of four to six weeks' recumbency would suffice. The interpubic ligaments heal by strong scar tissue when reduction of the symphysial diastasis is reasonably accurate. However, when there is a gap of over three-fifths of an inch (1.5 centimetres) there is likely to be interference with the function of the pelvic joints (Fig. 11).

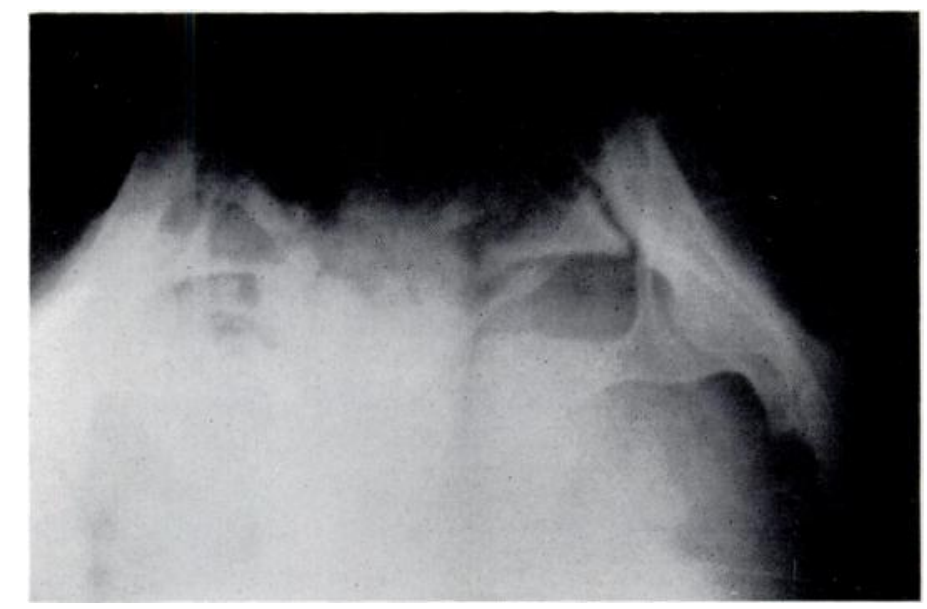

Fig. 8

Radiograph of sacrum, showing the reversed taper of the "keystone" of the pelvic arch.

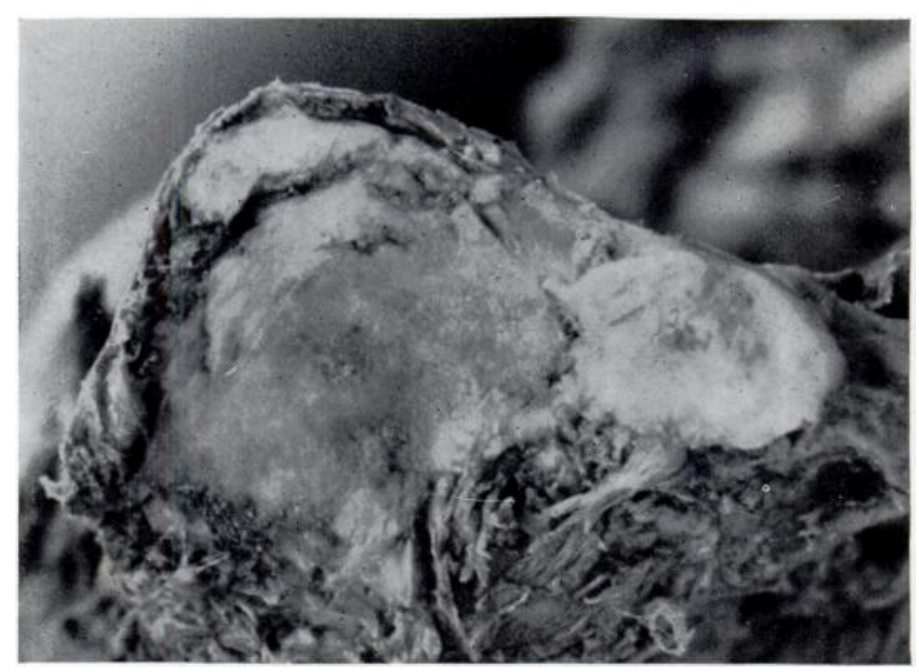

FIG. 9

The articular surface of the sacrum of an adult, showing degeneration of the articular cartilage.

If the hip is adducted, a force transmitted along the femoral shaft will produce a posterior dislocation, and the degree of both adduction and flexion will determine whether or not a fragment of bone is sheared from the acetabulum.

If the hip is flexed and abducted at the time of injury the acetabulum yields at the thinnest and weakest place and a central dislocation of the hip results (Fig. 12). To secure reduction it is necessary to apply force in an opposite direction to that which caused the fracture. The direction of the deforming force may sometimes be determined by finding out the position of the limbs before the patient was moved (Gissane 1958) or by study of the radiographs. Again, 
an appraisal of the lesion with the patient completely relaxed under general anaesthesia (Moore 1958) may be helpful.

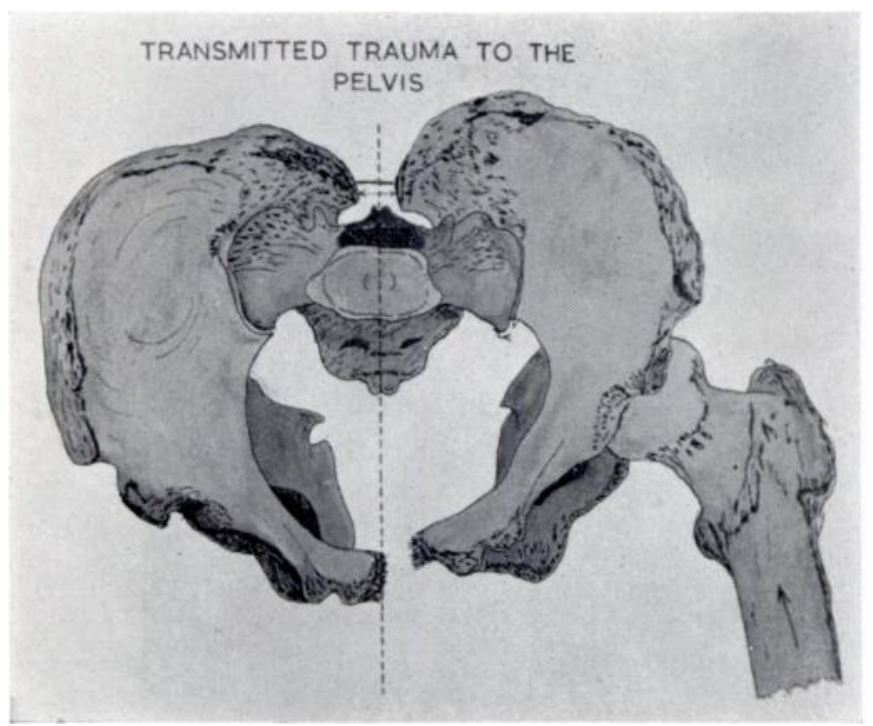

Fig. 10

Diagram of the lesion produced by a force transmitted along the shaft of the femur through the flexed hip.

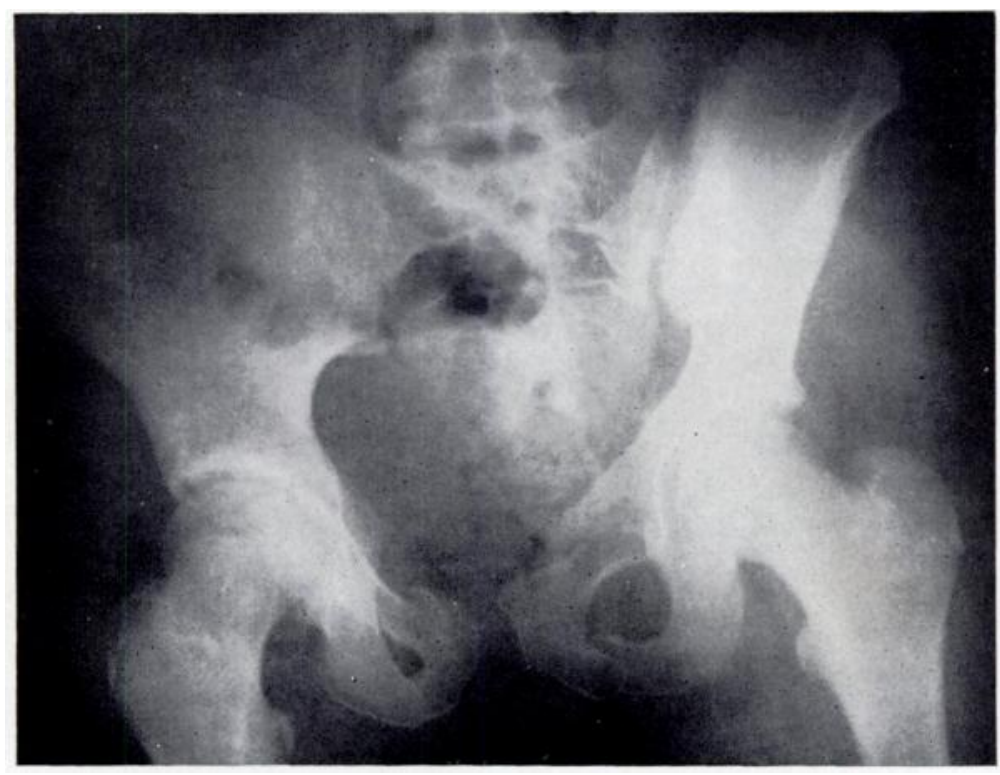

Fig. 11

Pelvic fracture caused by transmitted force in a European man aged nineteen years. Note the general similarity to the basic lesion shown in Figure 10.

From this study it appears that reduction can be achieved as long as two to three weeks after injury. On the other hand, associated injuries such as visceral damage may necessitate early operation. In that case the opportunity arises for reduction under direct vision. This opportunity should not be lost, because it is unlikely to occur again and because it makes possible the restoration of normal or nearly normal anatomy. It is likely that such anatomical

VOL. 42 B, No. 3, AUGUST 1960 
restoration might avoid late sequelae such as instability of the sacro-iliac joints and interference with child bearing (Figs. 13 and 14). For instance, reduction of the central dislocation shown in Figure 12 proved to be easy. It was effected by bimanual manipulation, one hand being introduced into the pelvic cavity through a transverse,

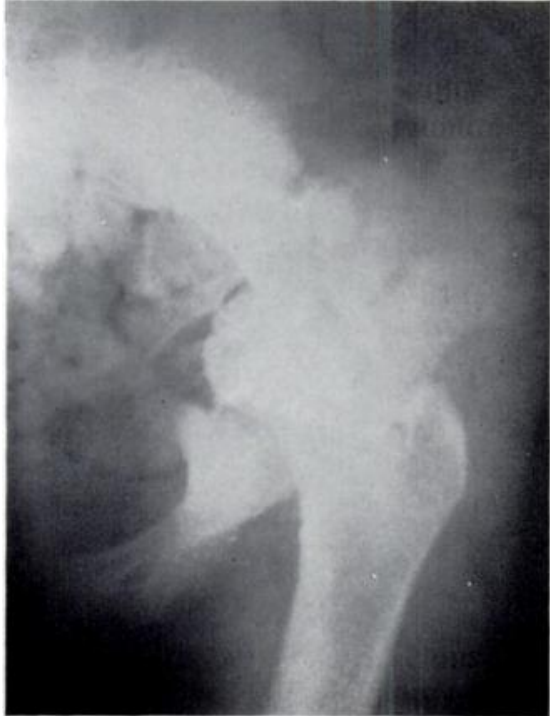

Fig. 12

Central dislocation of the hip in a European woman aged nineteen years.

It was associated with rupture of the urethra at the bladder neck Reduction of the fracture-dislocation was first attempted after the manner described by Ross (1957). This manipulative procedure consists in gradual, strong abduction and extension of the hip on the affected side. Reduction is effected by control of the pelvic fragment through the adductor

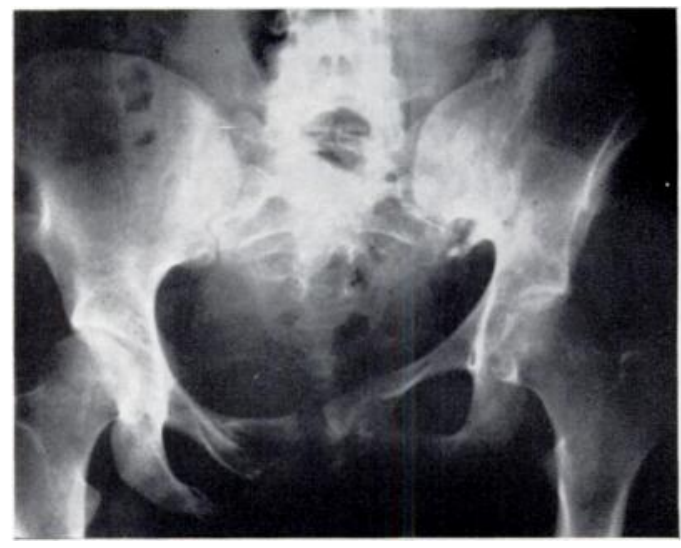

Fig. 13

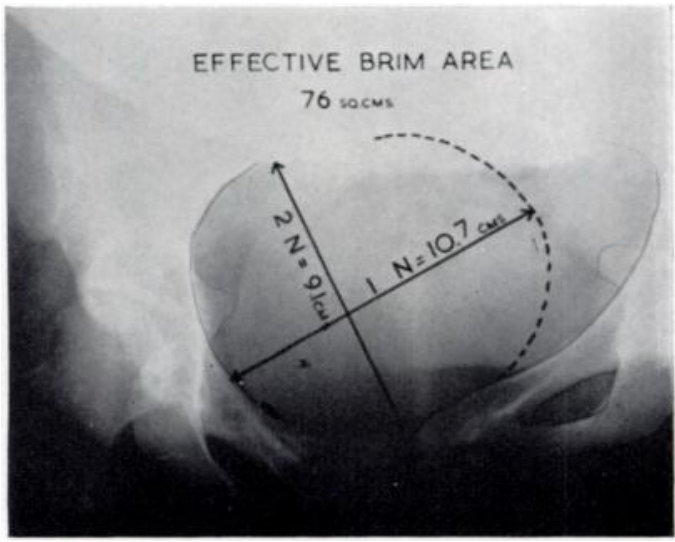

FIG. 14

The end result of a pelvic fracture-dislocation in a European woman aged twenty-one years. The effective area at the pelvic brim is $\mathbf{7 6}$ square centimetres. The area required for normal labour is 100 square centimetres.

and flexor muscles (Fig. 18). Reduction was incomplete (Fig. 19). The opportunity for reduction under direct vision afforded by the operative approach to the damaged urethra was then taken. A bimanual reduction was done. The intra-abdominal hand exerted firm pressure on the pubis (Fig. 20), and at the same time Ross's manoeuvre was performed with the other hand. There was an audible and palpable snap, and the reduction was complete. A short double plaster spica 


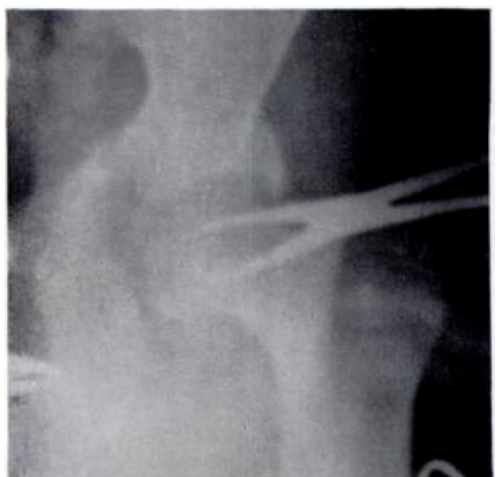

FIG. 15

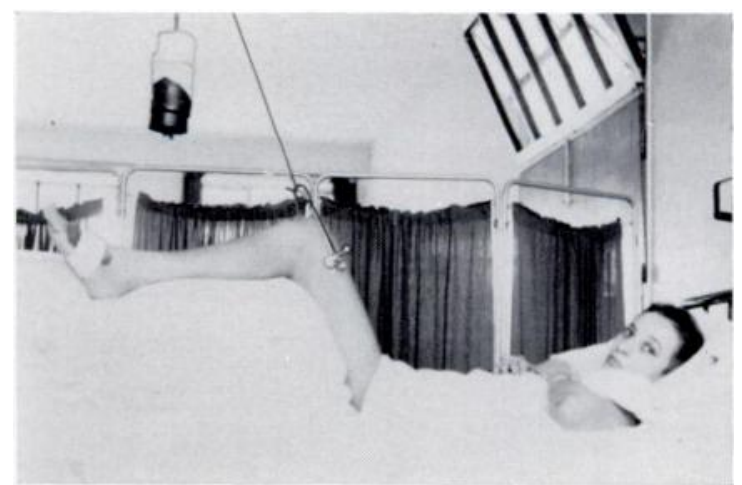

FIG. 16

Bimanual reduction of fracture-dislocation, and maintenance of reduction by skeletal traction applied through the flexed hip.

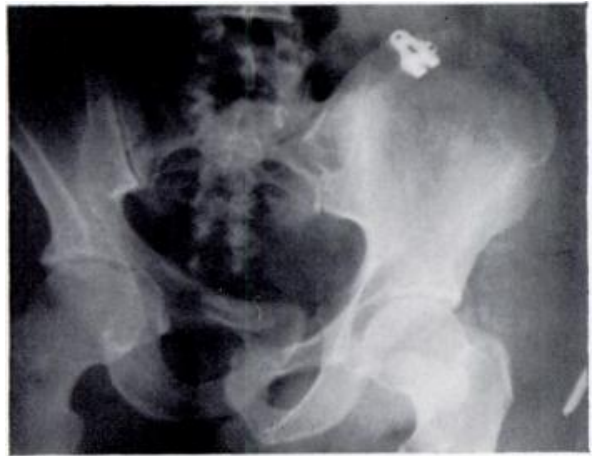

FIG. 17

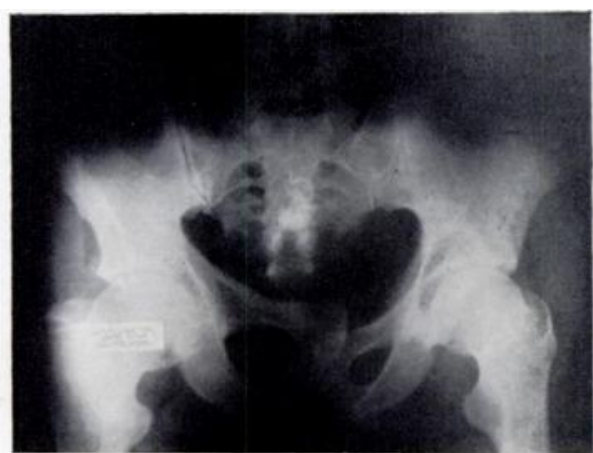

FIG, 19

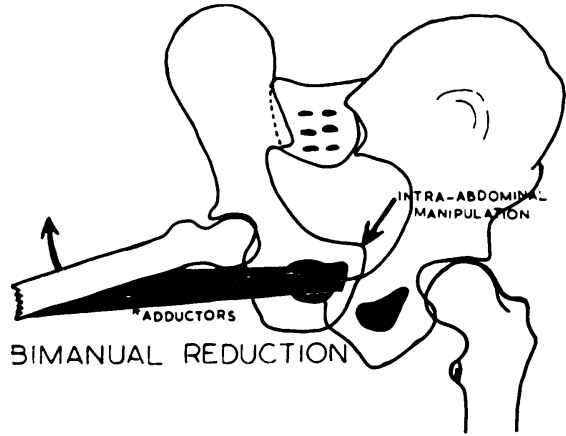

Fig. 20

Method of bimanual reduction used. Radiograph shows that complete reduction has been achieved.

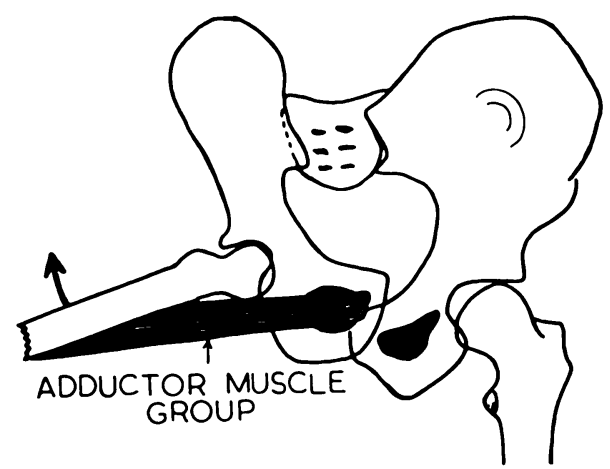

FiG. 18

Figs. 17 To 19

Compression injury of the pelvis caused by crushing between two vehicles. European man aged forty-six years. Attempted closed reduction by the method of Ross (1957). Incomplete reduction.

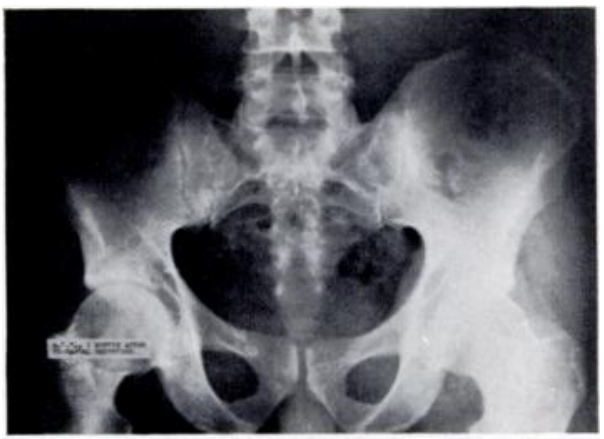

FIG. 21

VOL. 42 B, No. 3, AUgust 1960 
was applied and retained for two months. Complete anatomical restoration was secured (Fig. 21), but troublesome backache persisted for ten months after the patient started walking.

In the antero-posterior compression type of lesion (Fig. 22) there is wide diastasis at the symphysis pubis, and the sacro-iliac joint is opened on one or other side. This is an unstable lesion; it is caused by great violence, and is often associated with other severe injuries. Reduction of the pelvic displacement is easy; maintenance of the reduction is difficult. A plaster spica is not tolerated, and a pelvic sling is ineffective. Open reduction and internal fixation, on the other hand, may be carried out with little disturbance to the patient, and with immediate

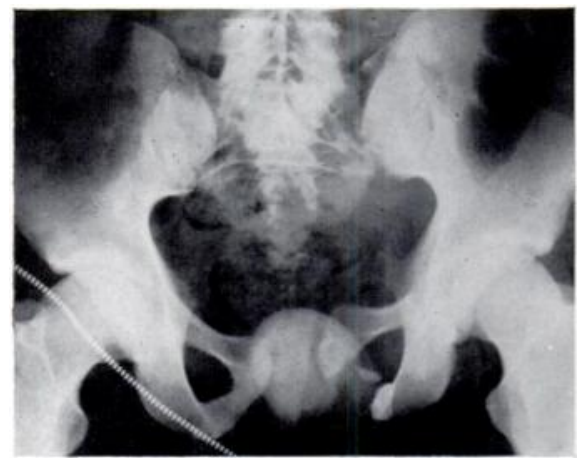

Fig. 22

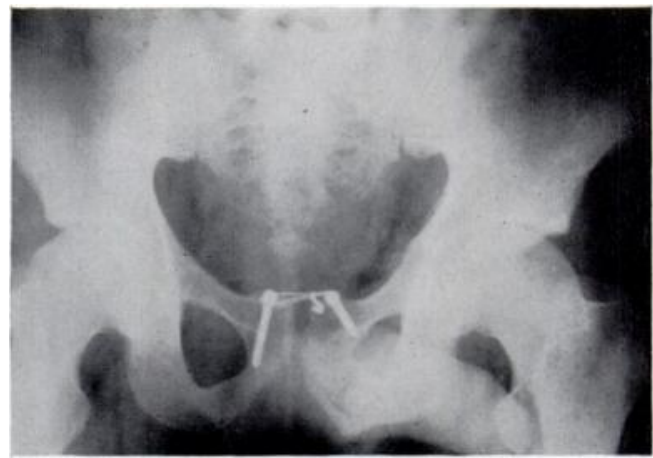

FIG. 23

Figure 22-European man aged twenty-one years. Fracture-dislocation of pelvis from antero-posterior compression. Figure 23-Radiograph showing position after bimanual reduction and fixation by screws and wire.

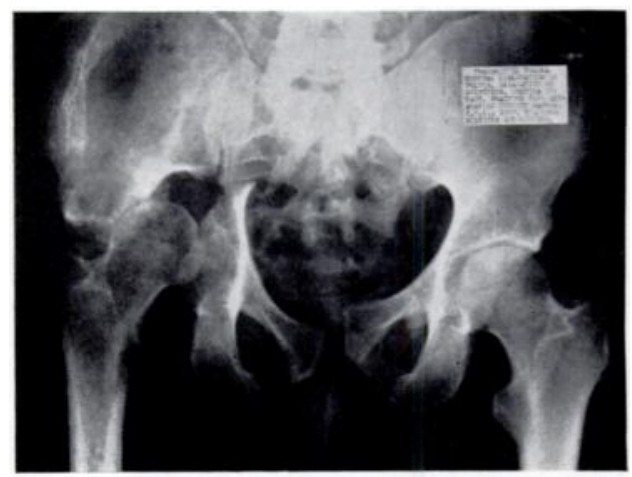

Fig. 24

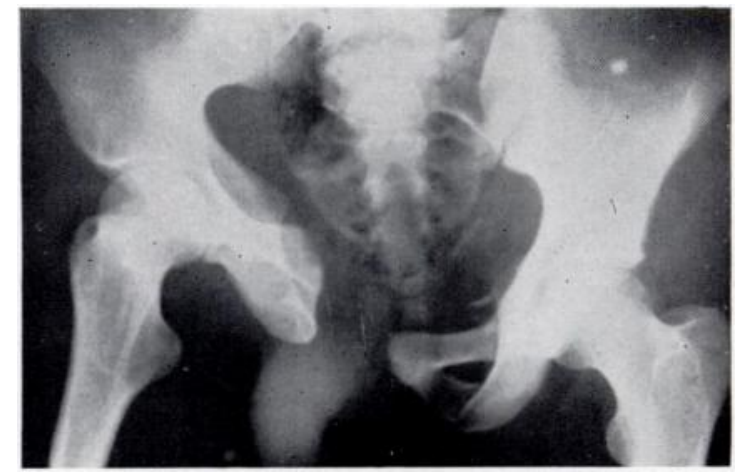

Fig. 25

Figure 24-European man aged forty-one years. Fracture-dislocation caused by combination of transmitted and direct violence. Note the disruption of the symphysis, central dislocation of the hip and fracture of the sacrum. Figure 25-European man aged twenty years. Fracture-dislocation caused by combination of transmitted and direct violence, which proved rapidly fatal.

and effective relief. Through a transverse lower abdominal incision the symphysis pubis is exposed extraperitoneally and the diastasis reduced by pressure on the sides of the pelvis. Internal fixation can be secured by inserting a two-inch screw into each pubic bone and lashing the screws together with stout wire (Fig. 23). If the urethra is damaged it is repaired at the same time. A short double plaster spica may be applied after two weeks, and retained for two to three months. In one case (Fig. 22) the pelvic injury was associated with a fracturedislocation of the lumbar spine and partial paraplegia. Reduction and fixation of the pelvic displacement were therefore deferred for three weeks; this delay did not prejudice the ease of the reduction, nor did it seem to affect the result. 
It will be clear that an accurate distinction between injuries caused by transmitted and those caused by direct violence is not always possible. Indeed, from the strength and duration of the violence there must in the extensive lesions be a combination of transmitted and direct force. The occupant of a vehicle which collides will be pitched forward so that his knee strikes resistance and transmits force to the pelvis. The compartment collapses and the victim is crushed, so that the pelvic injury may be complicated by the application of direct violence. Examples of such injuries are shown in Figures 24 and 25. In the first case (Fig. 24) the bony

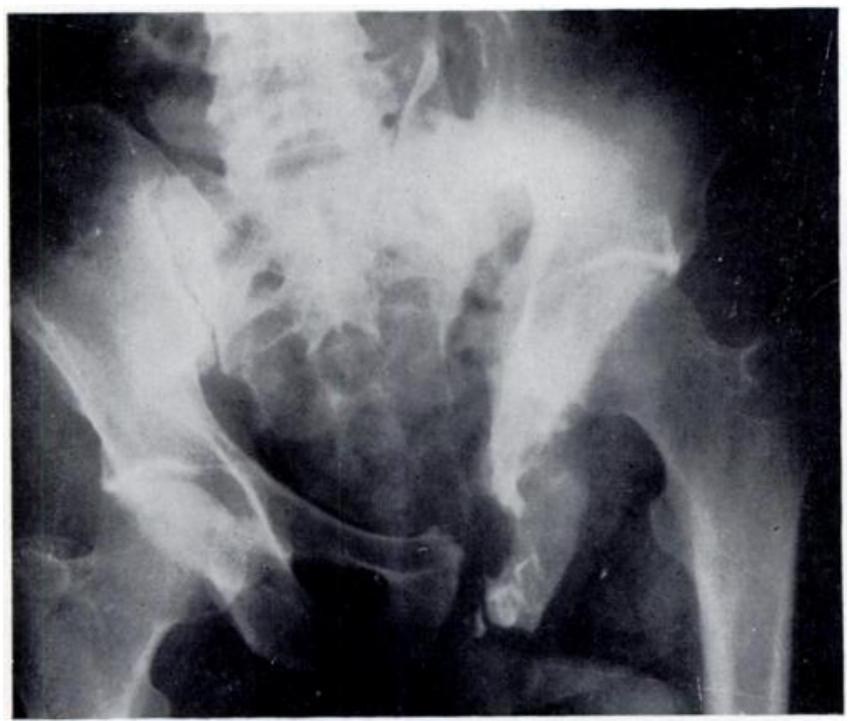

Fig. 26

Case 1-European man aged thirty-eight years. Radiograph shcwing result in an untreated case of fracture-dislocation of the peivis. Note the upward displacement of the left side of the pelvis.

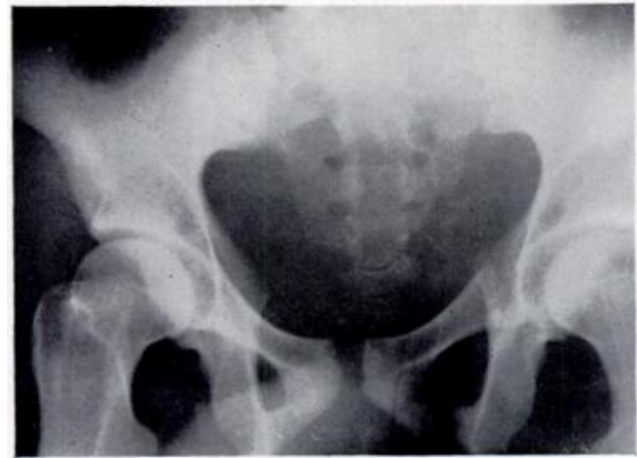

FIG. 27

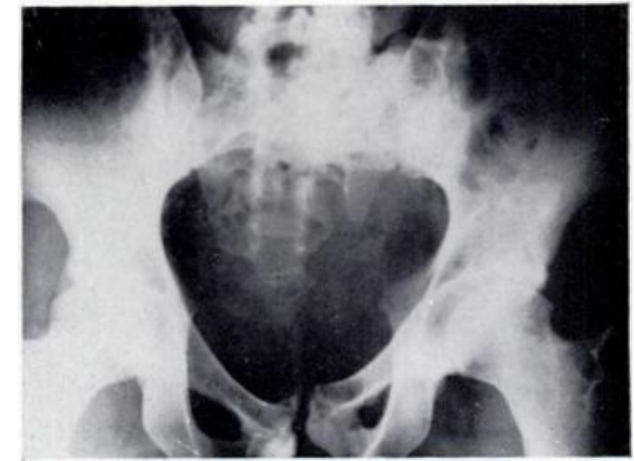

FIG. 28

Case 2. Figure 27-Fracture-dislocation of the pelvis in a European man aged twenty-two years. Untreated because of associated fracture-dislocation of the lumbar spine. Figure 28-Proximal displacement and lateral rotation of left ilium consequent on weight bearing.

injury was associated with a lesion of the first to the third sacral nerves on that side which proved irrecoverable. Open reduction failed here. The second patient (Fig. 25) died within a few hours of injury.

For comparison, examples are given of the results in cases in which accurate reposition was for various reasons not attempted or not achieved.

VOL. 42 B, No. 3, AUGUST 1960 
Case 1-A European man aged thirty-eight sustained extensive pelvic damage with rupture of the urethra. Repair of the urethral damage was undertaken but the patient refused later reduction of the pelvic displacement. One year after injury there was gross instability of the entire left wing of the ilium, which was displaced proximally and rotated laterally (Fig. 26). The limb "telescoped " one to two inches with each step, though the patient was able to walk with the aid of a stick. Backache was present, and the patient could not hurry or walk across uneven ground. It is possible that in this case disability could have been lessened by reduction and fixation of the pelvic injury undertaken at the same time as the urethral repair.

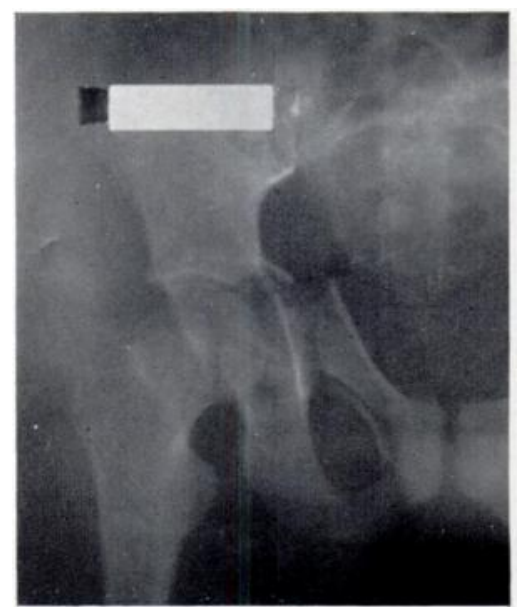

FIG. 29

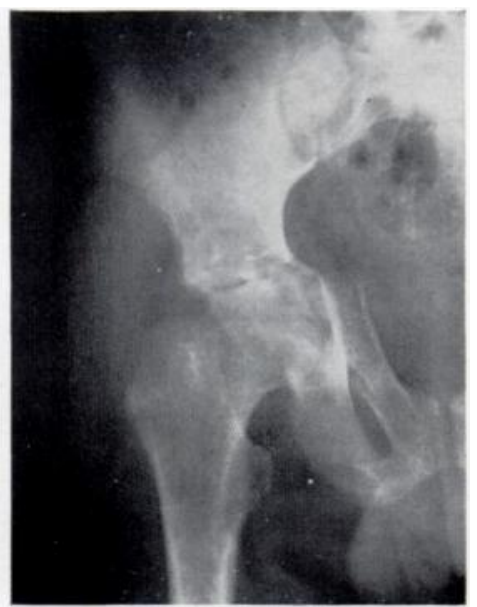

FIG. 30

Case 3. Figure 29-Central dislocation of the hip in a European man aged twenty-two years. Figure 30-Result after treatment by skin traction for three months. The hip was stiff and painful.

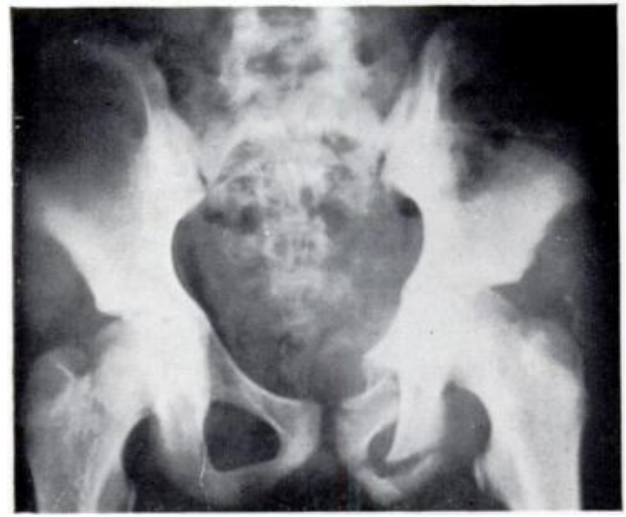

FIG. 31

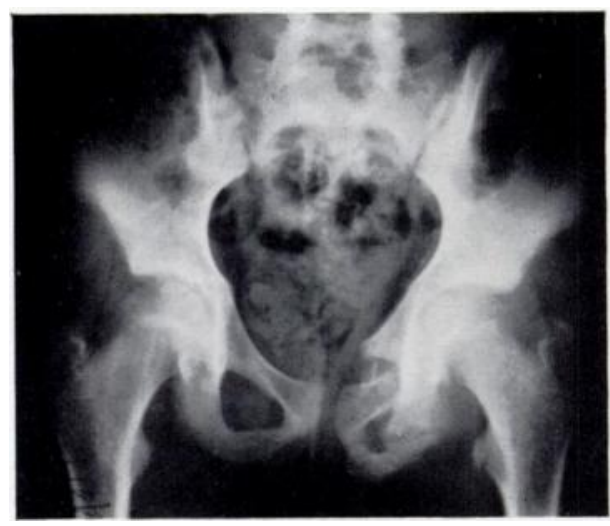

FIG. 32

Case 4. Figure 31-Fracture-dislocation of the pelvis in a European girl aged fourteen years. Note the fractures through the pelvic rami and the subluxation of the sacro-iliac joint. Figure 32Condition after weight bearing, showing increase of deformity and diminution of the width of pelvic brim.

Case 2-A European man of twenty-two sustained a pubic diastasis with fracture of both inferior pubic rami and with a vertical fracture through the left sacral foramina (Fig. 27). This was associated with a fracture-dislocation of the lumbar spine and accordingly remained untreated.

Weight bearing caused proximal displacement and lateral rotation of the left ilium with non-union of the sacral fracture and insecure fibrous ankylosis at the symphysis pubis (Fig. 28). Backache and inability to run were the sequelae.

Case 3-A European man of twenty-two sustained a central dislocation of the hip (Fig. 29). This was treated by three months' skin traction. The result was a painful stiff hip (Fig. 30). It is possible that in this case accurate reposition might have secured a better result. 
Case 4-A European girl of fourteen sustained fractures through the pubic rami, with lateral rotation of the part of the ilium bearing the acetabulum and with widening of the sacro-iliac joint on the affected side (Fig. 31). There was little general disturbance, and micturition was unaffected. The patient was told to remain in bed for six weeks, and then to get up. The result is shown in Figure 32. It would be acceptable in a man, but is probably not acceptable in a woman because of the permanent diminution in the size of the pelvic inlet.

\section{CONCLUSIONS}

1. The strength of the pelvic arch depends on the integrity of the anterior interpubic ligament, whose strength has been demonstrated by dissections. Once that ligament is divided the sacro-iliac ligaments offer little resistance to opening out of the pelvis.

2. The structure of the pelvis and hips is compared to an arcade formed by a central and two lateral arches. The weight of the trunk is transmitted to the lower limbs through this arcade. 3. Fractures of the pelvis are classified according to the mechanism of production. The case for anatomical reposition and internal fixation is stated, and case histories are given to illustrate the disabilities due to persistent deformity.

4. Reduction can be achieved as late as two or three weeks after injury. However, if early operation for visceral injury is necessary, there is a strong case for combining this with open reduction and fixation. In some cases the patient's general condition may preclude such procedures, but more usually the additional manipulations cause little additional operative shock and are fully justified by the subsequent increased comfort of the patient and the greater ease of nursing.

I wish to thank Miss E. Koch, my typist, for willing and efficient service; Professor C. L. Loubser of the Department of Forensic Medicine, University of Pretoria, for laboratory facilities; Drs F. W. McLachlan, A. v. B. Smith and A. Macdonald for radiological work, and $\mathrm{Mr} \mathrm{W}$. Gissane, Clinical Director of the Birmingham Accident Hospital, for his invaluable encouragement and advice. I record my thanks too to Miss J. Drake and Mr R. Gill, of the Birmingham Accident Hospital, for clerical and photographic work, and to Professor F. Geldenhuys, Dr F. N. Neser and Dr W. Schroeder of the Department of Gynaecology and Obstetrics, University of Pretoria, for permitting me to see the results of symphysiotomy. Figure 26 is reproduced by the courtesy of Mr C. L. Laubscher, Pretoria.

\section{REFERENCES}

Gissane, W. (1958): Personal communication.

MOORE, J. R. (1958): Personal communication.

Ross, W. T. (1957): Personal communication.

WATSON-Jones, Sir R. (1955): Fractures and Joint Injuries. Fourth edition, p. 940. Edinburgh and London: E. \& S. Livingstone Ltd.

vol. 42 B, NO. 3, AUgUST 1960 